\title{
The Effect of Probe Path Length Calibration on Dissolution Tests Performed with a Fiber-Optic In Situ Dissolution Test System
}

\author{
Pingping Xu, Xinxia Li*, Li Li, Yang Liu, Bin Kong, \\ Kun Nie, and Jian Chen \\ School of Pharmacy, Xinjiang Medical University, No.393 Xinyi Road, Xinjiang, Urumqi 830011, China
}

\begin{abstract}
The purpose of this research was to provide a method for calibrating the path length of probes used for dissolution tests performed with a fiber-optic dissolution test system (FODT). Methods found in pharmacopeia use the absorptivity of a 60.0 $\mu \mathrm{g} / \mathrm{mL}$ potassium dichromate solution for calibrating 10-mm cuvettes. This absorptivity should only be employed to calibrate probes with a 5-mm gap where the total path length is $10 \mathrm{~mm}$, since the calibration will be inaccurate if this absorptivity is used with different path length probes. In this article, we establish a calibration method using the absorptivities of potassium dichromate solutions at concentrations of $120.0,300.0$, and $600.0 \mu \mathrm{g} / \mathrm{mL}$ with $5-, 2-$, and $1-\mathrm{mm}$ cuvettes to calibrate probes with 2.0-, 1.0-, and 0.5-mm gaps, respectively. The calibration method established here for FODT minimizes calibration inaccuracies observed with calibration of probes using the method in Appendix IVA of the Chinese Pharmacopeia.
\end{abstract}

\section{INTRODUCTION}

D etermination of the release rate of active ingredients from drug preparations is an important procedure in pharmaceutical laboratories. Traditionally, the technique is time consuming and labor intensive as it requires the removal of samples at specified time points followed by analysis of the samples with UV-vis spectrophotometry or high performance liquid chromatography (HPLC). Fiber-optic dissolution test systems (FODT), first introduced in 1988 (1), make dissolution testing less time consuming and labor intensive; no sample withdrawal is required because measurements are carried out in situ using a fiber-optic probe inserted directly into each dissolution vessel. The use of FODT in the pharmaceutical industry is increasing because it is a more efficient approach for dissolution testing.

Several research groups (2-4) have worked to develop fiber-optic techniques for dissolution testing. Real time or rapid data collection is possible with FODT and provides a wealth of information including complete drug release profiles from dissolution tests. FODT is especially useful in the development of new drugs and new dosage regimes where a large number of drugs require dissolution screening. The principles of dissolution testing with FODT have been described in detail in previous reports $(5,6)$.

In FODT, the sample "volume" is simply the liquid present in the probe gap at the time of measurement. The path length is twice the length of this gap because there is a mirror at the probe tip that reflects the light back to the detector (5). By selecting a replaceable probe with an appropriate spacing, the path length can be varied. The path length of the probes must be calibrated before an

*Corresponding author.
FODT is used for dissolution testing. The calibration of an FODT is analogous to the calibration of a UV spectrophotometer. The absorptivity of a standard solution of potassium dichromate with a concentration of $60 \mu \mathrm{g} / \mathrm{mL}$ can be used to calibrate a 10-mm cuvette (7) or the path length of a 5-mm FODT probe. If the standard absorptivity is used to calibrate probes with different path lengths, inaccuracies can be introduced. In this study, probes with different path lengths were first calibrated using the potassium dichromate standard absorptivity cited in Appendix IVA of the Chinese Pharmacopeia (ChP). We defined this method as the Single-Parameter Method (SPM). The probes with different path lengths were then calibrated using the absorptivities of potassium dichromate obtained using a UV spectrophotometer with different standard concentrations and different path length cuvettes. We defined this method as the Multi-Parameter Method (MPM). After the probes were calibrated with these two methods, dissolution tests were performed with carbamazepine and hydrochlorothiazide tablets. Results from both calibration approaches were compared to determine the errors and validate calibration of the probes with the MPM.

The $C h P$ is not the only pharmacopeia to employ absorptivity for calculating the accumulative dissolution rates for certain drugs. This approach is also utilized by the British Pharmacopoeia (BP) for dissolution tests of phenylbutazone, chlortetracycline, and tylosin tablets, as well as for nonpharmacopeial methods $(8,9)$. The advantages of this approach are that it consumes less chemical standard and takes less time. However, the absorptivity is based on a specified concentration and a path length of $10 \mathrm{~mm}$. In this study, we employed an FODT to determine dissolution rates where detection was performed 
in situ and no sample dilution was required. Varying the path length to ensure that the absorbance is in the linear range is an approach that has been used in previous studies (10-12). When the absorbance of a sample exceeded the range of $0.2-0.8$ using a $10-\mathrm{mm}$ path length probe, we altered the probe path length according to the BeerLambert law so that the absorbance was within the linear range.

\section{MATERIALS AND METHODS Materials}

A fiber-optic drug dissolution in situ test system (FODT) was developed by Jian et al. (2) of the Xinjiang Medical University and Xinjiang FOCS Biotech Development Co., Ltd. A UV spectrophotometer (Cintra 40, GBC Scientific Equipment Pty. Ltd., Australia) was used for the offline control experiment.

Potassium dichromate standard reagents and carbamazepine tablets (100 and $200 \mathrm{mg}$ ) were purchased from Jiangsu Sihuan Bioengineering Co., Ltd. (Lot 0803041) and Beijing Nuohua Pharmaceutical Co., Ltd. (Lot x0303), respectively. Hydrochlorothiazide tablets $(50 \mathrm{mg})$ were purchased from Shandong Renhetang Yaoye Co., Ltd. (25 mg, Lot 080205). Carbamazepine (Lot 100142199503) and hydrochlorothiazide reference standards (Lot 100309-200702) were obtained from the National Institute for the Control of Pharmaceutical and Biological Products of China. Hydrochloric acid and sulfuric acid were all of analytical reagent grade and were obtained from Fuchen Chemical Reagents (Tianjin, China). Purified water was produced in the laboratory and degassed before use.

\section{Methods \\ Calibration of Different Path Length Probes with Potassium Dichromate}

A stock solution of potassium dichromate was prepared by dissolving $60 \mathrm{mg}$ of dried standard with $0.005 \mathrm{~mol} / \mathrm{L}$ sulfuric acid. Potassium dichromate solutions at 600.0, $300.0,120.0$, and $60.0 \mu \mathrm{g} / \mathrm{mL}$ were then prepared from the stock solution. The absorbances of the potassium dichro-

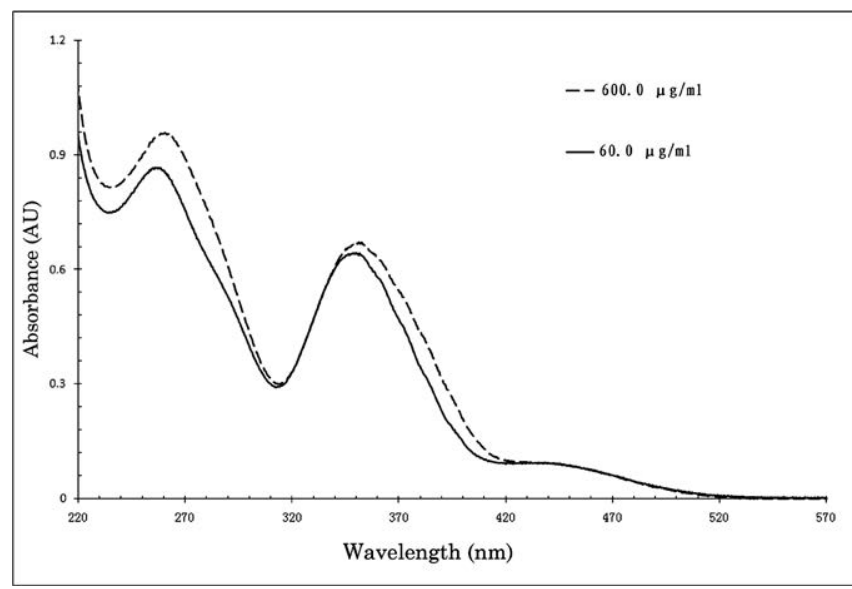

Figure 1. Absorption spectra of potassium dichromate at different concentrations.

mate solutions were determined using cuvettes with path lengths of $1,2,5$, and $10 \mathrm{~mm}$.

\section{Absorption Spectra of Potassium Dichromate Solutions \\ The absorption spectra of 60.0 and $600.0 \mu \mathrm{g} / \mathrm{mL}$ potas- sium dichromate solutions were determined from 220 to $440 \mathrm{~nm}$ using a UV spectrophotometer with $10-\mathrm{mm}$ and 1-mm cuvettes, respectively.}

\section{Dissolution Test of Carbamazepine Tablets}

Comparison of Errors after Calibration with SPM and MPM

Dissolution was carried out using Apparatus 2 (paddle) of the ChP at $150 \mathrm{rpm}$ and a constant temperature of $37 \pm 0.5^{\circ} \mathrm{C}$ using $1000 \mathrm{~mL}$ of diluted hydrochloric acid. The detection wavelength was $285 \mathrm{~nm}$, and the absorptivity was 518. Using FODT, probe gaps of 0.5 and $1.0 \mathrm{~mm}$ were used for carbamazepine tablets with label strengths of 200 and 100 mg, respectively. Sampling was performed at specified times. A control experiment was conducted using a UV spectrophotometer and the ChP method. The probe path lengths were calibrated before the dissolution testing using the SPM and MPM.

Table 1. Absorptivities of Different Concentrations of Potassium Dichromate at Different Wavelengths with Different Path Length Cuvettes $(n=6)$

\begin{tabular}{|c|c|c|c|c|c|c|c|c|}
\hline \multirow[b]{2}{*}{ Wavelength (nm) } & \multicolumn{2}{|c|}{$E_{10 m m}^{1 \%}(60.0 \mu \mathrm{g} / \mathrm{mL})^{a}$} & \multicolumn{2}{|c|}{$E_{5 m m}^{1 \%} \pm \% R S D(120.0 \mu \mathrm{g} / \mathrm{mL})$} & \multicolumn{2}{|c|}{$E_{2 m m}^{1 \%} \pm \% R S D(300.0 \mu \mathrm{g} / \mathrm{mL})$} & \multicolumn{2}{|c|}{$E_{1 \mathrm{~mm}}^{1 \%} \pm \% R S D(600.0 \mu \mathrm{g} / \mathrm{mL})$} \\
\hline & Average & Range & Average & Range & Average & Range & Average & Range \\
\hline 235 & 124.5 & $123.0-126.0$ & $126.9 \pm 0.09$ & $126.5-127.3$ & $134.0 \pm 0.13$ & $133.3-134.7$ & $135.9 \pm 0.16$ & $135.2-136.6$ \\
\hline 257 & 144.0 & $142.8-146.2$ & $147.3 \pm 0.07$ & 147.0-147.6 & $156.0 \pm 0.09$ & 155.4-156.6 & $158.4 \pm 0.21$ & 157.6-159.2 \\
\hline 313 & 48.6 & $47.0-50.3$ & $48.9 \pm 0.13$ & $48.7-49.1$ & $50.7 \pm 0.51$ & $50.4-51.0$ & $50.2 \pm 0.46$ & $49.9-50.5$ \\
\hline 350 & 106.8 & $105.5-108.5$ & $108.2 \pm 0.10$ & 108.0-108.4 & $112.4 \pm 0.10$ & $112.1-112.7$ & $111.0 \pm 0.18$ & $109.5-110.5$ \\
\hline
\end{tabular}

${ }^{a}$ Cited from ChP 
Table 2. Carbamazepine Tablets (100- and 200-mg) with FODT and Control Experiment (UV) Dissolution Data

\begin{tabular}{|c|c|c|c|c|c|c|}
\hline \multirow[b]{3}{*}{ Label Strength (mg) } & \multirow{2}{*}{\multicolumn{2}{|c|}{$\frac{\text { Single Parameter Method (SPM) }}{\text { Dissolution (\%) }(n=6)}$}} & \multicolumn{4}{|c|}{ Multi-Parameter Method (MPM) } \\
\hline & & & \multirow{2}{*}{$\Delta \bar{x}^{b}$} & \multicolumn{2}{|c|}{ Dissolution (\%) (n=6) } & \multirow{2}{*}{$\triangle \bar{x}$} \\
\hline & FODT $^{a}$ & Control & & FODT & Control & \\
\hline 100 & $79.7 \pm 4.1$ & $84.5 \pm 3.8$ & 4.8 & $83.7 \pm 1.9$ & $83.7 \pm 1.9$ & 0 \\
\hline 200 & $74.1 \pm 2.3$ & $82.0 \pm 0.4$ & 7.9 & $80.9 \pm 2.0$ & $82.9 \pm 1.0$ & 2.0 \\
\hline
\end{tabular}

a Path length was $2 \mathrm{~mm}$ for $100-\mathrm{mg}$ label strength and $1 \mathrm{~mm}$ for $200-\mathrm{mg}$ label strength.

${ }^{b}$ Absolute difference between the means

Absorptivities of Carbamazepine Solutions at Different Concentrations

Carbamazepine solutions of 19.9, 39.8, 99.6, and 195.2 $\mu \mathrm{g} / \mathrm{mL}$ were prepared, and their absorbances were determined using 10-, 5-, 2-, and 1-mm cuvettes, respectively, with a UV spectrophotometer.

\section{Dissolution Test of Hydrochlorothiazide Tablets}

Comparison of Errors after Calibration with SPM and MPM

Dissolution was carried out using Apparatus 1 (basket) of the ChP 2005 at $150 \mathrm{rpm}$ and a constant temperature of $37 \pm 0.5^{\circ} \mathrm{C}$ using $1000 \mathrm{~mL}$ of diluted hydrochloric acid. The detection wavelength was $272 \mathrm{~nm}$, and the absorptivity $(1 \mathrm{~cm}, 1 \%)$ of hydrochlorothiazide was 640 . Probes with 2.0-mm gaps were selected, and the dissolution test was carried out for $30 \mathrm{~min}$. At the end of the test, the control experiment was carried out according to the $\mathrm{ChP}$ method.

\section{Hydrochlorothiazide Absorptivities at Different}

Concentrations

Hydrochlorothiazide solutions of 10.8, 21.6, 54.0, and $108.0 \mu \mathrm{g} / \mathrm{mL}$ were prepared, and the absorbances were measured using 10-, 5-, 2-, and 1-mm cuvettes, respectively, with a UV spectrophotometer.

\section{RESULTS}

\section{Potassium Dichromate Absorptivities and Absorption Spectra}

The absorptivities of the potassium dichromate solutions at different concentrations and wavelengths are listed in Table 1 and are significantly different at different concentrations with different cuvette path lengths ( $p<$ 0.05). The absorption spectra of 60.0 and $600.0 \mu \mathrm{g} / \mathrm{mL}$ potassium dichromate solutions (Figure 1) show that the absorbance maxima and minima are different at these two concentrations.

\section{Dissolution Testing of Carbamazepine Tablets}

Dissolution Errors after Calibration with SPM and MPM

The dissolution test results for $60 \mathrm{~min}$ are shown in Table 2. The standard deviations (SD) for carbamazepine tablets of $100 \mathrm{mg}$ and $200 \mathrm{mg}$ were 4.8 and 7.9 , respectively, when the probes were calibrated with SPM. When the probes were calibrated with MPM, the SD decreased
Table 3. Absorptivities of Carbamazepine Solutions with Different Concentrations

\begin{tabular}{lcc}
\hline Concentration $(\boldsymbol{\mu g} / \mathbf{m L})$ & Path Length $(\mathbf{m m})$ & Absorptivity \\
\hline 19.9 & 10.0 & 517.2 \\
\hline 39.8 & 5.0 & 519.6 \\
\hline 99.6 & 2.0 & 539.1 \\
\hline 195.2 & 1.0 & 559.3 \\
\hline
\end{tabular}

Table 4. Actual $\left(C_{1}\right)$ and Calculated $\left(C_{2}\right)$ Concentrations ${ }^{a}$ with Different Cell Path Lengths

\begin{tabular}{lcccc}
\hline $\boldsymbol{C}_{\mathbf{1}}(\boldsymbol{\mu g} / \mathbf{m L})$ & Path Length, L $(\mathbf{m m})$ & $\boldsymbol{C}_{\mathbf{1}} \times \mathbf{L}$ & $\mathbf{A}$ & $\boldsymbol{C}_{\mathbf{2}}(\boldsymbol{\mu g} / \mathbf{m L})$ \\
\hline 9.95 & 10 & 99.5 & 0.5146 & 9.93 \\
\hline 19.91 & 5 & 99.55 & 0.5173 & 19.97 \\
\hline 49.8 & 2 & 99.6 & 0.5365 & 51.8 \\
\hline 97.6 & 1 & 97.6 & 0.5459 & 105.4 \\
\hline
\end{tabular}

${ }^{a}$ Absorptivity (518) given in the ChP for carbamazepine was used.

significantly to 0.0 and 2.0 for carbamazepine tablets of $100 \mathrm{mg}$ and $200 \mathrm{mg}$, respectively $(p<0.05)$.

\section{Absorptivities of Carbamazepine Solutions at Different Concentrations}

The absorptivities of carbamazepine solutions at concentrations of $19.9,39.8,99.6$, and $195.2 \mu \mathrm{g} / \mathrm{mL}$ were calculated according to the Beer-Lambert law (Table 3). In dissolution tests with FODT, the final concentrations for carbamazepine tablets with label strengths of 100 and $200 \mathrm{mg}$ were 100 and $200 \mu \mathrm{g} / \mathrm{mL}$, respectively. Therefore, based on the results in Table 3, absorptivities of 539.1 and 559.3 should be employed to calculate the dissolution rate of carbamazepine 100- and 200-mg tablets, respectively. The results were inaccurate when the absorptivity for carbamazepine cited in the ChP (518) was used to calculate the dissolution rates for the carbamazepine 100- and 200-mg tablets (Table 4). If the absorptivity is constant, the absorbance should remain constant when the concentration is increased and the path length is decreased. However, the absorbance was not constant (Table 4), indicating that the absorptivity is not constant. 
Table 5. Dissolution Data for Hydrochlorothiazide Tablets with FODT (path length $4 \mathrm{~mm}$ ) and Control Method (UV)

\begin{tabular}{|c|c|c|c|c|c|}
\hline \multicolumn{3}{|c|}{ Single Parameter Method (SPM) } & \multicolumn{3}{|c|}{ Multi -Parameter Method (MPM) } \\
\hline \multicolumn{2}{|c|}{ Dissolution (\%) $(n=6)$} & \multirow{2}{*}{$\triangle \bar{x}^{a}$} & \multicolumn{2}{|c|}{ Dissolution (\%) $(n=6)$} & \multirow{2}{*}{$\triangle \bar{x}$} \\
\hline FODT & Control & & FODT & control & \\
\hline $91.5 \pm 1.9$ & $94.8 \pm 1.8$ & 3.3 & $91.0 \pm 6.8$ & $92.4 \pm 6.5$ & 1.6 \\
\hline
\end{tabular}

${ }^{a}$ Absolute difference between the means

Table 6. Absorptivities of Hydrochlorothiazide Solutions at Different Concentrations

\begin{tabular}{lcc}
\hline Concentration $(\boldsymbol{\mu g} / \mathbf{m L})$ & Path Length $(\mathbf{m m})$ & Absorptivity \\
\hline 10.8 & 10.0 & 644 \\
\hline 21.6 & 5.0 & 650 \\
\hline 54.0 & 2.0 & 666 \\
\hline 108.0 & 1.0 & 670 \\
\hline
\end{tabular}

Table 7. Actual $\left(C_{1}\right)$ and Calculated $\left(C_{2}\right)$ Concentrations ${ }^{a}$ with Different Cell Path Lengths

\begin{tabular}{lcccc}
\hline $\boldsymbol{C}_{\mathbf{1}}(\boldsymbol{\mu g} / \mathbf{m L})$ & Path Length, L $(\mathbf{m m})$ & $\boldsymbol{C}_{\mathbf{1}} \times \mathrm{L}$ & $\mathbf{A}$ & $\boldsymbol{C}_{\mathbf{2}}(\boldsymbol{\mu g} / \mathbf{m L})$ \\
\hline 10.8 & 10 & 108 & 0.6957 & 10.87 \\
\hline 21.6 & 5 & 108 & 0.7019 & 21.93 \\
\hline 54.0 & 2 & 108 & 0.7196 & 56.22 \\
\hline 102.0 & 1 & 102 & 0.6951 & 108.6 \\
\hline
\end{tabular}

${ }^{a}$ Absorptivity cited in the ChP for hydrochlorothiazide was used.

\section{Dissolution Test of Hydrochlorothiazide Tablets}

Dissolution Errors after Calibration with SPM and MPM

A control experiment was carried out according to the $\mathrm{ChP}$ method. The dissolution test results for $30 \mathrm{~min}$ are listed in Table 5. The SDs between the control and FODT dissolutions with calibration of the probes carried out by SPM and MPM were 3.3 and 1.6, respectively.

\section{Absorptivities of Hydrochlorothiazide Solutions at Different} Concentrations

The absorptivities of hydrochlorothiazide solutions with concentrations of $10.8,21.6,54.0$, and $108.0 \mu \mathrm{g} /$ $\mathrm{mL}$ were calculated according to the Beer-Lambert law (Table 6). For the dissolution test of 25-mg hydrochlorothiazide tablets, the final concentration was $25 \mu \mathrm{g} /$ $\mathrm{mL}$. Therefore, according to the absorptivity results, an absorptivity of 650 should be used to calculate solution concentrations. However, the absorptivity was not constant when the concentration was increased and the path length was decreased correspondingly. Inaccurate results would be generated if the dissolution concentrations were calculated with an absorptivity of 640 as cited in the ChP method (Table 7).

\section{DISCUSSION}

Chemical deviations from Beer's law can occur when the absorbing species are involved in an equilibrium reaction. In potassium dichromate solutions, the following equilibrium reaction occurs:

$$
\mathrm{Cr}_{2} \mathrm{O}_{7}^{2-}+\mathrm{H}_{2} \mathrm{O} \Leftrightarrow 2 \mathrm{H}^{+}+2 \mathrm{CrO}_{4}^{2-}
$$

At low potassium dichromate concentrations, the primary ion is $\mathrm{HCrO}_{4}$, with approximately $1-4 \%$ of the $\mathrm{Cr}_{2} \mathrm{O}_{7}{ }^{2-}$ ion formed by dimerization where the amount of $\mathrm{Cr}_{2} \mathrm{O}_{7}{ }^{2-}$ depends on the chromium concentration (13). The absorptivities of the $\mathrm{HCrO}_{4}{ }^{-}$ion $\left(E_{1 \mathrm{~cm}}^{1 \%}\right)$ are $124.5,144.0$, 48.6, and 106.6 at 235, 257, 313, and $350 \mathrm{~nm}$, respectively. When the concentration of the solution of potassium dichromate is high, the dimer $\mathrm{Cr}_{2} \mathrm{O}_{7}{ }^{2-}$ is formed. The spectral absorptivity of this $\mathrm{Cr}_{2} \mathrm{O}_{7}{ }^{2-}$ dimer is similar to that of $\mathrm{HCrO}_{4}^{-}$, but its overall spectral shape is significantly different, as shown in Figure 1 (14).

The Beer-Lambert law is valid only for low concentrations of analyte. This is because absorbances will change as the refractive index varies with analyte concentration. For sufficiently low concentrations of analyte, the refractive index remains constant, hence the Beer-Lambert law is valid.

The accumulative dissolution rates (\%) of different drugs as determined with probes calibrated using the Single Parameter and Multi-Parameter methods show that the results from the probes calibrated by the Single Parameter method had larger mean absolute errors than the results from the probes calibrated by the Multi-Parameter method. Furthermore, the mean absolute errors decreased as the probe path length increased. Therefore, we suggest that when employing FODT, the probe path length should be calibrated using the absorptivities of potassium dichromate at the corresponding concentrations. When drug concentrations exceed a specified range where the Lambert-Beer law is valid, the absorptivity should not be employed to calculate the accumulated dissolution. In this case, calibration with a reference substance should be employed to ensure generation of accurate data.

\section{ACKNOWLEDGMENTS}

The authors would like to acknowledge Xinjiang FOCS Biotech Co., Ltd., China, for its contribution to this work. This research was supported by the Project of Xinjiang 
Key Laboratory of Molecular Biology and Endemic Diseases (No. XJDX0208-2011-04) and the National Natural Science and Technology Foundation of China (No. 81260486).

\section{REFERENCES}

1. Josefson, M.; Johansson, E.; Torstensson, A. Optical Fiber Spectrometry in Turbid Solution by Multivariate Calibration Applied to Tablet Dissolution Testing. Anal. Chem. 1988, 60 (24), 2666-2671.

2. Chen, C. S.; Brown, C. W. A Drug Dissolution Monitor Employing Multiple Fiber Optic Probes and a UV/ Visible Diode Array Spectrophotometer. Pharm. Res. 1994, 11 (7), 979-983.

3. Li, W.; Chen, J.; Xiang, B.; An, D. Simultaneous on-line dissolution monitoring of multicomponent solid preparations containing vitamins $B_{1}, B_{2}$, and $B_{6}$ by a fiberoptic sensor system. Anal. Chim. Acta 2000, 408, 39-47.

4. Zhu, B.; Xing, J. F.; Chen, J. Monitoring the dissolution test of Metronidazole tablets by fiber-optic chemical sensor in situ system. Acta Pharm. Sin. 1994, 29, 369-374.

5. Nie, K.; Li, L.; Li, X. X.; Zhang, Y.; Mu, X.; Chen, J. Monitoring Ambroxol Hydrochloride SustainedRelease Tablets Release by Fiber-Optic Drug Dissolution In Situ Test System. Dissolution Technol. 2009, 16 (1), 14-17.

6. Nie, K.; Li, L.; Li, X. X.; Geng, D.; Zhang, Q.; Tuo, M.; Xu, P.; Chen, J. In Situ Fiber-Optic Dissolution Assisted by a Mathematical Separation Model of Dynamic ThreeWavelength K-Ratio Spectrophotometry. Dissolution Technol. 2010, 17 (2), 15-18.
7. Pharmacopoeia of the People's Republic of China; Pharmacopeia Committee of the Ministry of Public Health; People's Medical Publishing House: Beijing, 2005.

8. Schatz, C.; Ulmschneider, M.; Altermatt, R.; Marrer, S.; Altorfer, H. Manual In Situ Fiber Optic Dissolution Analysis in Quality Control. Dissolution Technol. 2000, 7 (2), 6-12.

9. Schatz, C.; Ulmschneider, M.; Altermatt, R.; Marrer, S.; Altorfer, $\mathrm{H}$. Thoughts on Fiber Optics in Dissolution Testing. Dissolution Technol. 2001, 8 (2), 6-11.

10. Ding, T. M.; Zhang, Z. X.; Chen, J.; Li, X. X.; Xiong, H. J. Fiber Optic Sensor Process Analysis on Dissolution Rate of Aminophylline Tablets. Chinese J. Hosp. Pharm. 2005, 25 (7), 604-606.

11. Yao, J.; Zou, H.; Li, X. X.; Chen, J. Processing Analysis on Dissolution Rate of Doxycycline Hyclate Tablets. J. Xinjiang Med. U. 2003, 26 (6), 525-526.

12. Xu, P. P.; Li, X. X.; Tuo, M. F.; Chen, J. Study on the Effect of Temperature on the Dissolution Test of Metformin Hydrochloride Tablets with Fiber-Optic In Situ Dissolution System. Northwest Pharm.l J. 2010, 25 (4), 281-283.

13. Burke, R. W.; Mavrodineanu, R. Certification and Use of Acidic Potassium Dichromate Solutions as an Ultraviolet Absorbance Standard-SRM 935. National Bureau of Standards Special Publication 260-54; Office of Standard Reference Materials; U.S. Government Printing Office: Washington, D.C., 1977.

14. Clare, J. F. Calibration of UV-vis spectrophotometers for chemical analysis. Accredit. Qual. Assur. 2005, 10 (6), 283-288. 